\title{
Majority Ordering and the Morphological Pattern Spectrum
}

\author{
Alessandro Ledda and Wilfried Philips \\ Ghent University, Telin-IPI, St. Pietersnieuwstraat 41, B-9000 Gent, Belgium, \\ ledda@telin.UGent.be, \\ WWW home page: http://telin.ugent.be/ ledda/
}

\begin{abstract}
Binary and grayscale mathematical morphology have many applications in different area. On the other hand, colour morphology is not widespread. The reason is the lack of a unique ordering of colour that makes the extension of grayscale morphology to colour images not straightforward. We will introduce a new majority sorting scheme that can be applied on binary, grayscale and colour images. It is based on the area of each colour or grayscale present in the image, and has the advantage of being independent of the values of the colours or grayvalues. We will take a closer look at the morphological pattern spectrum and will show the possible differences of the morphological pattern spectrum on colour images with the grayscale image pattern spectrum.
\end{abstract}

\section{Introduction}

\subsection{Binary Morphology}

Mathematical morphology [1] [2] is based on set theory. The shapes of objects in a binary image are represented by object membership sets. Object pixels have value 1 , the background pixels have value 0 . Morphological operations can simplify image data, preserving the objects' essential shape characteristics, and can eliminate irrelevant objects.

Binary mathematical morphology is based on two basic operations, defined in terms of a structuring element (short: strel), a small window that scans the image and alters the pixels in function of its window content: a dilation of set $A$ with strel $B(A \oplus B)$ enlarges the object (more 1-pixels will be present in the image), an erosion $(A \ominus B)$ lets it shrink (the number of 1-pixels in the image diminishes) (see figure 1).

Mathematically, the basic operators are defined as:

$$
\begin{aligned}
& \text { dilation: } A \oplus B=\bigcup_{b \in B} T_{b}(A) \\
& \text { erosion : } A \ominus B=\bigcap_{b \in B} T_{-b}(A)
\end{aligned}
$$

with $T_{b}(A)$ the translation of set $A$ over vector $b$. This formulation can be rewritten as equation 2 , used for grayscale morphology.

Other operations, like the opening (an erosion followed by a dilation) and the closing (a dilation followed by an erosion), are derived from the basic operators. 


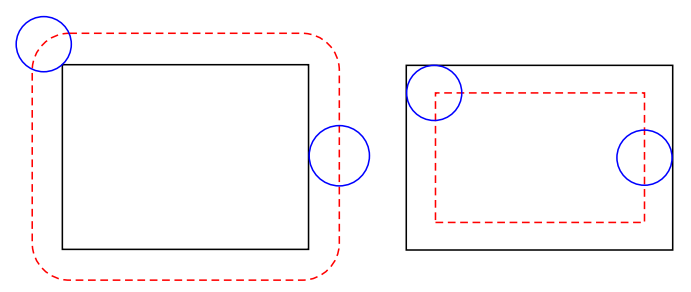

Fig. 1. The basic morphological operators. Solid line: original object; Dashed line: result object; Circle: structuring element. Left: dilation; Right: erosion.

\subsection{Grayscale Morphology}

The morphological theory can be extended to grayscale images with the threshold approach or with the umbra approach [2]. The latter permits the use of grayscale structuring elements but the resulting grayvalues after a morphological operation can be outside the original range. We will use the threshold approach.

For binary images, the union and intersection operation are used for the dilation and erosion, respectively. In the case of grayscale images (sets become functions), the union and intersection of sets are replaced by the maximum and minimum of grayvalues. For the dilation and erosion we now get:

$$
\begin{aligned}
& \text { dilation }:(f \oplus g)(a)=\max \{f(b) \mid a-b \in g, b \in f\} \\
& \text { erosion }:(f \ominus g)(a)=\min \{f(b) \mid b-a \in g, b \in f\}
\end{aligned}
$$

where $f$ is the function representing the grayscale image and $g$ is the structuring element.

\subsection{Colour Morphology}

A colour image is represented in some colour space. The colours in such a colour space can be interpreted as vectors.

A frequently used space is the RGB space, used in computer systems. There are three colour bands (red, green and blue) that together represent the colours of the pixels in the image in the RGB-space.

There is no absolute ordering of the colour vectors, so the max- and minoperation can't be extended easily to vectors and therefore an extension to colour morphology is not straightforward. In order to be able to extent the principles of grayscale morphology to colour images, the colours in a colour image have to be ordered in some way [3] [4]. A way of doing this is to transform the vectors into scalars with a lexicographical ordering scheme, further explained in section 2.1. We also present a new technique (section 2.3) that not only can be applied to colour images, but also to binary and grayscale images.

\subsection{The Pattern Spectrum}

If we take a strel and use it to perform an opening on a binary image, some objects will disappear. If we take a bigger strel, then more elements in the image 
will vanish. In this way we can determine how the number of eliminated objects increases when the image is morphologically opened using strels $n B=B \oplus$ $B \oplus \ldots \oplus B$ ( $n$ times) of increasing size $n$. The resulting plot of the number of eliminated pixels versus the strel size $n$ is called the pattern spectrum (PS) [5].

The pattern spectrum is a histogram of the distribution of the sizes of various objects displayed in an image. Formally, it is defined as follows:

$$
P S(A ; B)(n)=\sharp[(A \circ n B)-(A \circ(n+1) B)], n \geq 0
$$

where $\circ$ is the opening symbol and $\sharp$ is the count of pixels. Note that $0 B=\{0\}$.

Notice that a different strel results in different pattern spectra, and thus in other values for the parameters.

The same equation can be used for grayscale images. The difference with the binary case is the count of pixels that is replaced by a count of decrease in grayvalues.

\section{Methodology}

\subsection{Colour Morphology}

First, we transform the RGB image into an HSL image. In the HSL space the colour channels are the luminance (the intensity), the saturation (the purity), and the hue (the primary colour).

We will use the double-cone HSL representation. The luminance $L$ has values between 0 and 1 , the hue $H$ lies between $0^{\circ}$ and $360^{\circ}$. The saturation $S$ has values between 0 and 1 for $L=\frac{1}{2}$ and the maximum $S$ decreases linearly as $L$ goes to 0 or 1 . At $L=0$ and $L=1$ the saturation can only be 0 .

The ordering of the colours is done with a lexicographical ordering rule on the HSL-vector [4]. Putting $L$ as a criterion on the first line in the lexicographical ordering ( $L$-ordering) gives a different ordering than using $S$ or $H$ on that line. In this paper we will use the lexicographical rules from [4] (the $H$-ordering uses a saturation-weighted hue).

The comparison of the hue values is the comparison between the acute angles of the hue values with a reference hue $H_{0}$. The choice of $H_{0}$ can be arbitrary or calculated in function of the image content (section 2.1).

Vector Ordering According to [4], a morphological operation can be performed on an a colour image (in HSL space) by comparing the current pixel with the other pixels in the window of the structuring element. This operation can be quite intensive because a lot of comparisons have to be made. To avoid the recalculations of the order of one pixel compared to another, it is better to transform the HSL image into a scalar image, based on the lexicographical ordering.

A 24 bits RGB image will then be transformed into a 24 bits "grayscale" image. The most important rule in the lexicographical ordering occupies the highest 8 bits, the least important rule the lowest 8 bits. Each pixel gets some value between 0 and $2^{24}-1$. As a result, equation 3 can be used. 
Origin of Hue A possible value for $H_{0}$ could be the hue that appears the most in the image. If the background is the most present in the image, then all hue values are referenced to the hue of the background.

We can also take the hue value of the average chromaticity vector as $H_{0}$ (also in [6]). To calculate this average, we transform each hue value, represented as a point on a circle with an angle $\theta$ and with radius $r=1$, in its Cartesian coordinates. Then we average the vectors and transform this average vector back to polar coordinates. This gives us $H_{0}$ : it is the average angle $\bar{\theta}$.

An alternative way to calculate the average hue value is to take the histogram of the hue in the image and then give the radius $r$ the value of the number of pixels with a certain hue. This approach is less accurate because the histogram consists of a certain number of bins, thus introducing a limited number of possible hue values, while the hue originally could have any value in its domain. This is not really a problem because for the vector ordering (section 2.1) the hue was also made discrete.

A less elegant method for the definition of the average hue is to perform a number of shifts of the hue histogram with a number of bins, because of the periodic character of the hue domain. The introduced shift is needed because, for example, a hue of $10^{\circ}$ and $350^{\circ}$ would give an average of $180^{\circ}$, while we would expect $0^{\circ}$. If it is shifted with $10^{\circ}$, then the hue angles will be $20^{\circ}$ and $0^{\circ}$, so the average hue is $10^{\circ}$ and minus the shift this would give us the correct result. We calculate the variance of every shifted spectrum, and finally calculate the average hue (using the same formulation as for the mean object size for the pattern spectrum (section 2.2)) from the histogram with the smallest variance. In the example, the variance of this shifted spectrum is smaller than the variance of the original.

\subsection{The Pattern Spectrum}

In the binary case, the pattern spectrum is a graph of how many pixels were removed because of the opening of the image with the increasing strel $(n+1) B$.

By extension, the pattern spectrum of a grayscale image tells how many of grayscale intensity disappeared. This is the sum of the decrease in grayvalue for each pixel. The number of graylevels gives an indication how dark the resultant image becomes after an opening.

In the case of colour images, the interpretation is more abstract. The principle is the same as the one for grayscale images, but we could now speak of a decrease in colour intensity. The pattern spectrum gives an indication of the change in colour after an opening. This change depends on the ordering used. For example, in the case of an ordering with saturation in the first ordering rule, the pattern spectrum values indicate how the saturation decreases.

From the pattern spectrum we can extract different parameters [5], [7] that provide statistical information about the content of the image:

- Mean object size: the mean strel size or mean area; 
- Average roughness: the entropy, a quantification of the shape-size complexity;

- Normalised average roughness: the entropy divided by $\log _{2}\left(N_{\max }+1\right)$;

- B-shapiness: a quantitative measure of the resemblance of the objects to the strel shape;

- Maximal n $\left(N_{\max }\right)$ : the last bin (the highest $n$-value, when all image objects are sieved out) of the pattern spectrum histogram.

\subsection{Majority Ordering by Total Area}

In this section we propose a new type of sorting of the pixels, the majority sorting scheme (MSS). Instead of using the grayvalues or ordered colours, we count the number of pixels present in the image for each colour. All morphological operations are then performed on the newly obtained image, as if it is a grayscale image. See figure 2 for a visual example.
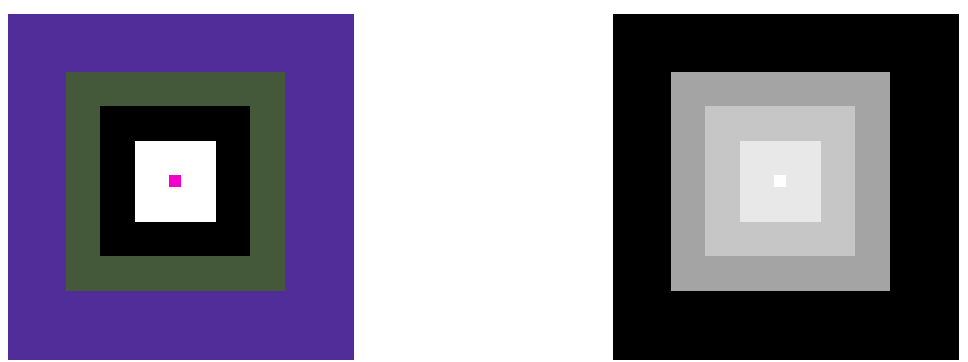

Fig. 2. Left: colour image; Right: MSS ordering map.

The motivation comes from the fact that in general the background is most present in the image, that important colours (objects) are highly present and that details or noise are rare colours. These assumptions are at the same time the restrictions of this technique.

Colours with the same number of pixels will be treated equally, but it is possible to steer the area values by adding a constant to it, for example if a specific background colour has to be chosen. For the pattern spectrum we will treat such colours equally.

With the MSS, the pattern spectrum gives an indication of the change in colour (or grayvalue), where the least dominant colours disappear first.

A technical difficulty is the fact that quantisation of the number of colours or grayvalues is necessary. If too many different colours are present, then too many equal small areas will be detected, which cancels the effect of the area ordering. Therefore, in most cases a quantisation will have to be done, but not too drastic so that the useful colour differences in the image would be retained.

A suggested quantisation is to reduce the number of colours (for example using peer group filtering [8]) until this number is the same as (or a certain percentage of) the number of different levels in the majority sorted image. 
Notice that the background has the biggest area, so the erosion and dilation operations should be switched, or the values of the areas must be switched.

This technique has several advantages:

- The technique can be used for binary, grayscale and colour images; also an extension to multispectral images is possible with the same majority ordering scheme;

- Colour images with only two colours will be treated as if it were binary images;

- The technique doesn't expect a specific colour space to work with;

- We don't have to define a value $H_{0}[6]$;

- The technique is quasi invariant for colour and grayscale transformations (e.g. $\gamma$-compensation); when all colours change in a new map of unique colours (the transformation is bijective), then there is no difference in the majority ordering;

- Using only one RGB colour band of a grayscale image will give the same results as the grayscale image itself.

\section{Experimental}

\subsection{Objects of One Colour}

If all objects in the image have the same colour, then the image can be seen as a binary image. Morphological operations will produce the results we expect with the majority sorting scheme and the lexicographical orderings. The pattern spectra are the same shape, so the spectral parameters will be identical.

There are some exceptions, though. In the case of the $S$ - and $H$-ordering, the comparison of the luminances (see [4]) is referenced to $L=\frac{1}{2}$, which means that there is no difference between colours with $L$ lying the same distance from $L=\frac{1}{2}$. Another effect can occur with the $H$-ordering: the ranking of the background colour can be higher than that of the object colour. The object on a background is then regarded as a hole in an object. The choice of $H_{0}$ is important in this case. These effects are not present in the case of the pattern spectrum of the majority ordered image.

\subsection{Objects of More Colours}

Figure 3 shows two objects with a small edge. This could be the effect of blurring of the image.

The majority sorted spectrum shows a peak at a lower bin, which indicates pixels that don't specifically belong to a big object. In this case these are the pixels from the blurring ring.

The $L$-ordered spectrum shows pixels that disappear after an opening with a $13 \times 13$ square strel. These are the values between the edges. This kind of feature can make the interpretation of the pattern spectrum difficult. 


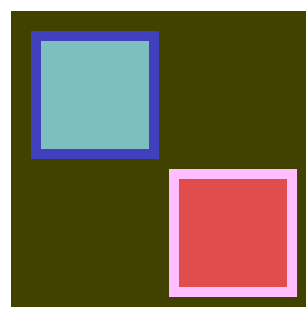

(a) Original image

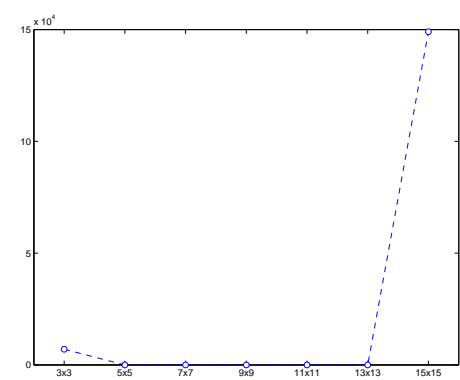

(b) PS (majority ordered)

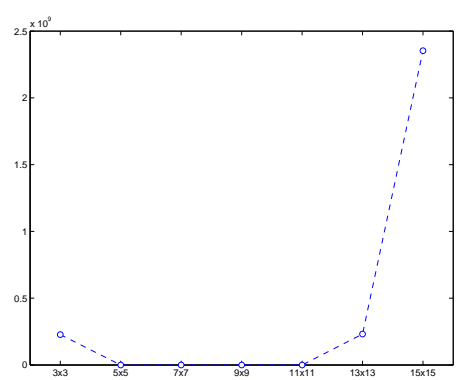

(c) PS ( $L$-ordered)

Fig. 3. Different pattern spectra for an artificial colour image.

\subsection{Real Images}

Figure 4(a) shows a medical image. The pattern spectrum of the majority sorted image shows the most realistic graph (figure 4(c)). There are no objects 160 times the strel (a square with side 3 ) present, so the $L$-ordering doesn't produce a useful spectrum. The same problem is present with the other lexicographical orderings.

\section{Conclusion}

Grayscale morphology can be used on colour images, if the colours are ordered in some way. The use of the morphological pattern spectrum for colour images can be a big advantage in situations where important colour information is lost when the colour image is transformed to grayscale.

This ordering can be done with a lexicographical rule. But we also proposed a majority sorting scheme that orders the colours according to the count of pixels of that colour present in the image. This method has some advantages over the lexicographical ordering scheme.

\section{References}

1. Serra, J.: Image Analysis and Mathematical Morphology. Volume 1. Academic Press, New York (1982) 


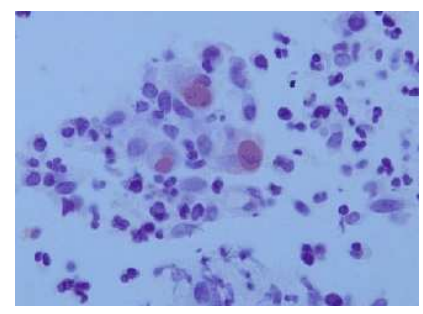

(a) Original

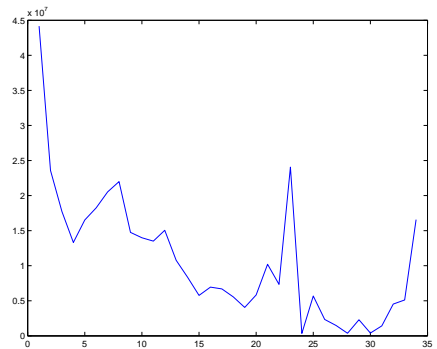

(c) PS with majority ordering

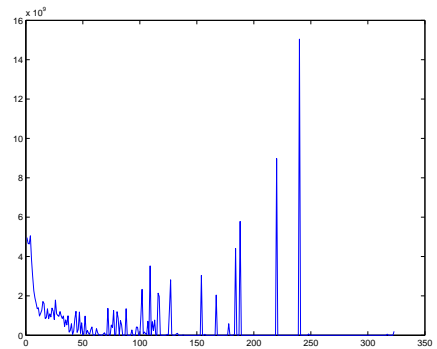

(e) PS with $S$-ordering

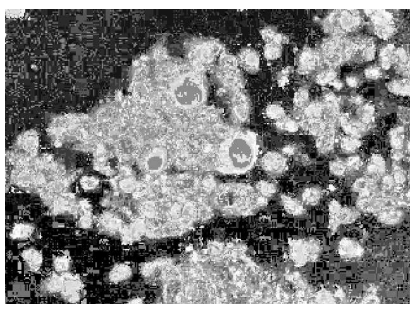

(b) MSS ordered map

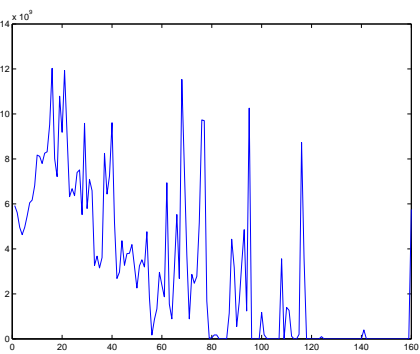

(d) PS with $L$-ordering

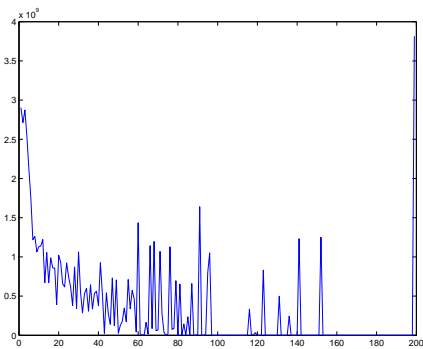

(f) PS with $H$-ordering

Fig. 4. Different pattern spectra for a colour image.

2. Haralick, R., Shapiro, L.: 5. In: Computer and Robot Vision. Volume 1. AddisonWesley (1992)

3. Peters II, R.: Mathematical morphology for angle-valued images. In: Proceedings of the SPIE, Nonlinear Image Processing VIII. Volume 3026. (1997) 84-94

4. Hanbury, A., Serra, J.: Mathematical Morphology in the HLS Colour Space. In: BMVC 2001, Manchester, UK (2001) 451-460

5. Maragos, P.: Pattern Spectrum and Multiscale Shape Representation. IEEE Transactions on Pattern Analysis and Machine Intelligence 11 (1989) 701-716

6. Hanbury, A.: Lexicographical order in the HLS Colour Space. Technical Report N04/01/MM, Centre de Morphologie Mathématique Ecole des Mines de Paris (2001)

7. Banerjee, S., Sahasrabudhe, S.: C-factor: a morphological shape descriptor. Journal of Mathematical Imaging and Vision 4 (1994) 43-55

8. Deng, Y., Kenney, C., Moore, M., Manjunath, B.: Peer group filtering and perceptual color image quantization. In: Proceedings of IEEE International Symposium on Circuits and Systems. Volume 4. (1999) 21-24 